\title{
Modeling nanostructure evolution using temperature-dependent radiolysis and kinetics of nanoscale reactions in liquid cell TEM
}

\author{
Serin Lee ${ }^{1}$, Nicholas Schneider ${ }^{2}$, Shu Fen $\operatorname{Tan}^{3}$ and Frances Ross ${ }^{3}$ \\ ${ }^{1}$ MIT, Cambridge, Massachusetts, United States, ${ }^{2}$ Renata Global, United States, ${ }^{3}$ MIT, United States
}

Over the last several years, the technique of liquid cell transmission electron microscopy (TEM) has been developed and refined for imaging liquid samples with good spatial and temporal resolution. The most exciting aspect of liquid cell TEM is that it provides us with a way to complete the triangle of structure-properties-processing of materials under controlled conditions that include temperature, electrochemical biasing, and liquid composition [1,2]. Temperature control is particularly important as temperature is a key parameter in liquid phase processes such as the operation of battery materials and other electrochemical phenomena such as corrosion and etching, as well as being a useful variable in understanding the physics of crystal growth, nanostructure evolution, and self-assembly $[3,4]$.

Here, we will discuss the effect of temperature on the process of beam-induced solution-phase crystal growth in silver, a well-studied phenomenon [5] Temperature is expected to influence several aspects of this process including radiolysis, diffusion, and nucleation. We focus on obtaining a quantitative view of the effect of temperature on radiolysis, aiming to build a robust model to calculate the equilibrium concentration of chemical species in the liquid medium under electron beam irradiation as a function of temperature.

In liquid cell TEM, the irradiating electrons affect the chemistry of the suspending medium, typically an aqueous solution, producing molecular and radical products such as hydrogen, oxygen, and hydrated (solvated) electrons and reducing metal ions in solution to create metal nanoparticles. First, we address the behavior of the pure water solvent radiolysis species as a function of temperature using a kinetic model [6] with temperature-dependent parameters. Temperature-dependent G values were used, and the reaction rates of species with known activation energy and the Arrhenius coefficient were scaled according to Arrhenius behavior. Figure 1 shows how this model predicts temperature effects on the radiolysis-driven equilibrium concentrations. The temperature dependence of the steady-state concentration of the radiolysis products in pure water is plotted in Figure 1a (the dose rate used was $7.5^{*} 10^{7} \mathrm{~Gy} / \mathrm{s}$, typical TEM conditions at $300 \mathrm{keV}$, beam of $1 \mu \mathrm{m}$ radius and $1 \mathrm{nA}$ current). The color-coded map of Figure $1 \mathrm{~b}$ has dose rate and temperature as variables. It shows the ratio of the steady-state concentrations of two key representative reducing and oxidizing species - the hydrated electron and hydroxyl radical - again in pure water. The red area shows the region where the environment is expected to be reducing, corresponding to circumstances where we might anticipate nanocrystal growth from metal cations in solution. In contrast, the blue area shows the region where the environment is expected to be more oxidizing, which may lead to the etching and dissolution of any existing metal nanoparticles. It can be seen that at fixed dose rate, increasing the temperature can change the solution from reducing to oxidizing, and that increasing temperature has a similar effect to decreasing the dose rate.

Next, we explored the effect of temperature on aqueous salt solution radiolysis and beam-induced growth of metal nanocrystals. The model includes the complete radiolysis reaction set for the complete set of chemical species in the initial solution. As an example, we consider the metal ion Ag+ and the conjugate ion NO3-. Several previous studies separately added the processes of metal cation reduction [7] or anion reaction [8] to a pure water system to study the redox chemistry inside a liquid cell TEM. 
However, in order to fully understand the interaction between the radiolysis species, metal cations, and anions, the kinetic model should incorporate both of the reactions as all reactions are interlinked. The temperature-dependent steady-state concentration of radiolysis products including silver cations and reduced silver are shown in Figure 1c. The steady-state concentration of reduced silver increases as temperature increases at fixed-dose rate. At $7.5^{*} 10^{7} \mathrm{~Gy} / \mathrm{s}$, when the temperature is increased from $20 \mathrm{C}^{\circ}$ to $40 \mathrm{C}^{\circ}$, the steady-state concentration of reduced silver is doubled. At fixed temperature, increasing the reduced silver concentration by this factor would require changing the dose rate by about in order of magnitude at room temperature, as calculated using the same model system. The calculation, therefore, suggests that both the temperature and dose rate are important parameters affecting the radiolysis chemistry in the liquid medium. This complete model provides an opportunity to understand how radiolysis species behave at different temperatures under the combined effect of other important experimentally controllable variables for liquid cell experiments: as well as dose rate and temperature, these are the initial concentration of the solution, $\mathrm{pH}$, and aeration. By adding these real-world parameters, an N-dimensional 'phase diagram' can be built and used as a map to predict the effects, perhaps with broader use for other researchers across the field.

We will finally show the results of testing this model by comparison of calculated results with experimental observations. The data is derived from measurements of nanoparticle generation rates as well as the beam-induced etching of metal thin films at different temperatures. We are excited by the opportunities presented by liquid cell TEM to develop and test a robust model that can provide steps towards enabling temperature to be used quantitatively in liquid cell experiments, with applications in probing the physics of nanostructure evolution, energy storage materials, corrosion, and catalyst synthesis [9].

(a)

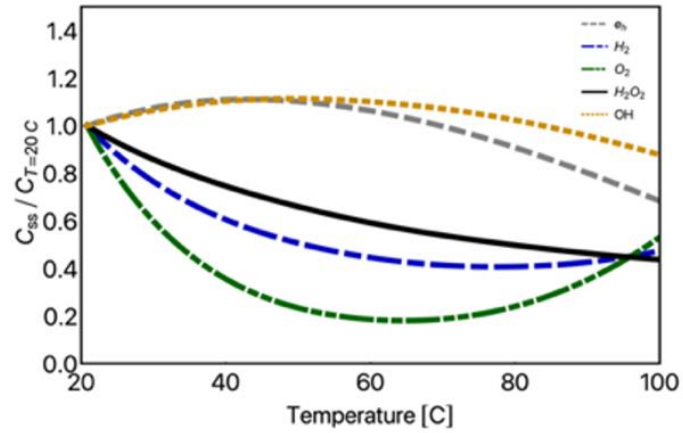

(b)

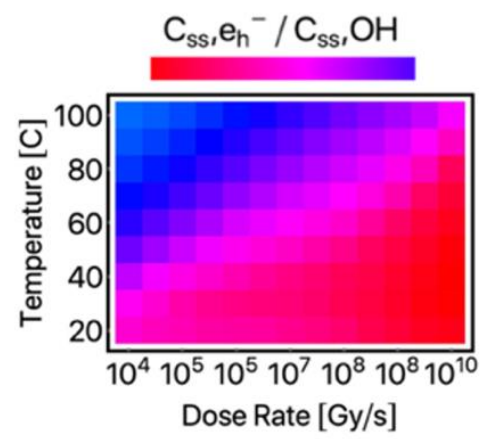

Figure 1. (a) Steady-state concentrations $\left(\mathrm{C}_{\mathrm{ss}}\right)$ of radiolysis products as functions of temperature for pure, deaerated water. (b) Color-coded map showing the ratio of the $\mathrm{C}_{\mathrm{ss}}$ of reducing and oxidizing agent in pure, deaerated water as a function of dose rate and temperature. Dose rate used for plotting was both $7.5 * 10^{7} \mathrm{~Gy} / \mathrm{s}$. 


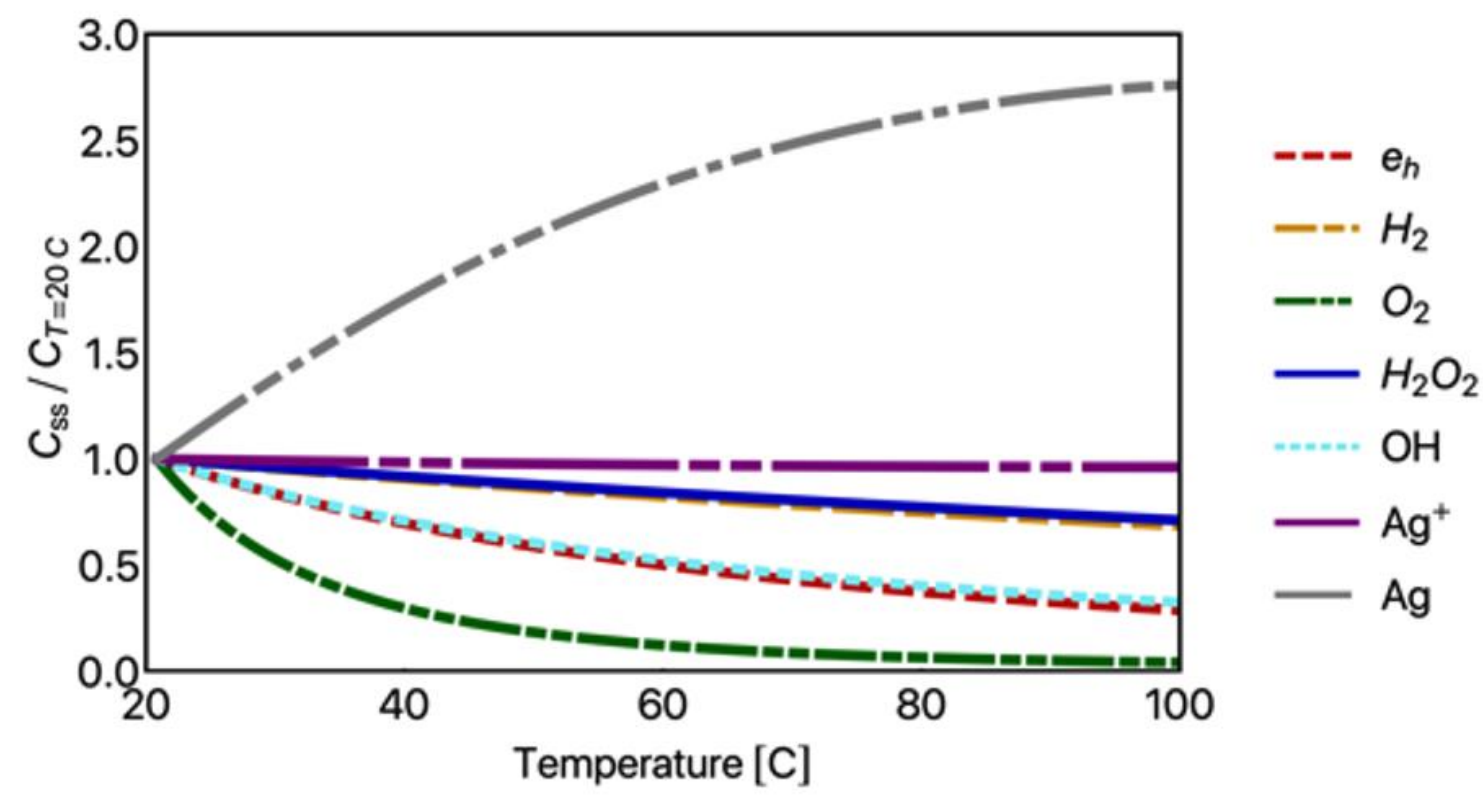

Figure 2. $\mathrm{C}_{\mathrm{ss}}$ of radiolysis products as functions of temperatures for $0.01 \mathrm{M}$ silver nitrate aqueous solution. Dose rate used for plotting was both $7.5^{*} 10^{7} \mathrm{~Gy} / \mathrm{s}$.

References

[1] Ross, F. M. Opportunities and challenges in liquid cell electron microscopy. Science 350, aaa9886 (2015)

[2] Hwang, S., Chen, X., Zhou, G. \& Su, D. In Situ Transmission Electron Microscopy on Energy-Related Catalysis. Adv. Energy Mater. 10, 1-24 (2020)

[3] Tan, S. F. et al. Real-Time imaging of Nanoscale Electrochemical Ni Etching under Thermal Conditions. Accepted

[4] Ma, Shuai, et al. Temperature effect and thermal impact in lithium-ion batteries: A review. Prog. Nat. Sci.28.6 (2018): 653-666a

[5] Woehl, Taylor J., et al. "Direct in situ determination of the mechanisms controlling nanoparticle nucleation and growth." ACS nano 6.10 (2012): 8599-8610.

[6] Schneider, N. M. et al. Electron-Water interactions and implications for liquid cell electron microscopy. J. Phys. Chem. C 118, 22373-22382 (2014)

[7] Park, J. H. et al. Control of Electron Beam-Induced Au Nanocrystal Growth Kinetics through Solution Chemistry. Nano Lett. 15, 5314-5320 (2015)

[8] Ambrožič, B. et al. Controlling the radical-induced redox chemistry inside a liquid-cell TEM. Chem. Sci. 10, 8735-8743 (2019).

[9] This work made use of facilities and instrumentation supported by NSF through the Massachusetts Institute of Technology Materials Research Science and Engineering Center under Grant DMR1419807. Serin Lee acknowledges funding from The MIT Energy Initiative Seed Fund. 